\title{
Técnicas nucleares y su versatilidad para la utilización y estudio de diversos procesos.
}

\author{
Esquivel, Alexander \\ Centro de Investigaciones Hidráulicas e Hidrotécnicas - (CIHH). Universidad Tecnológica de Panamá. \\ Panamá, Panamá \\ alexander.esquivel@utp.ac.pa / ORCID:0000-0003-4782-1335 \\ Kodama, Yasko \\ Instituto de Pesquisas Energéticas e Nucleares \\ São Paulo, Brasil \\ ykodama@ipen.br \\ Villarreal, José \\ Instituto de Innovación Agropecuaria de Panamá \\ Panamá, Panamá \\ jevilla38@gmail.com \\ Juri Ayub, Jimena \\ Instituto de Matemática Aplicada San Luis - (IMASL). Universidad Nacional de San Luis \\ San Luis, Argentina \\ jjuri@unsl.edu.ar \\ González, Guadalupe \\ Centro Regional de Chiriquí. Universidad Tecnológica de Panamá. \\ David, Panamá \\ guadalupe.gonzalez1@utp.ac.pa \\ Tejedor-Flores, Nathalia \\ Centro de Investigaciones Hidráulicas e Hidrotécnicas - $(\mathrm{CIHH})$. Universidad Tecnológica de Panamá. \\ Panamá, Panamá \\ nathalia.tejedor@utp.ac.pa
}

\section{Abstract}

The study of various topics of science is carried out using tools that often do not achieve the depth of analysis required to respond to situations that affect the world in general, are often invasive and pollute. Over time it has been shown that nuclear techniques are 
a viable alternative to develop more comprehensive studies in various fields of science such as: health, agriculture, industry, environment, security, among others. This group work session was organized considering the need to inform society about the other side of nuclear energy and its positive contributions to the development of countries, to make them more competitive and environmentally friendly.

Keywords: Nuclear techniques, isotopes, tracers, radiopharmaceuticals, energy.

\section{Resumen}

El estudio de diversos tópicos de la ciencia se efectúa haciendo uso de herramientas que muchas veces no logran la profundidad de análisis requerido para dar respuesta a situaciones que afectan el mundo en general, muchas veces son invasivos y contaminantes. Con el pasar del tiempo se ha demostrado que las técnicas nucleares son unas alternativas viables para desarrollar estudios más completos en diversos campos de la ciencia, por ejemplo: Salud, sector agropecuario, industria, medio ambiente, seguridad, entre otros. Está mesa de trabajo se organizó viendo la necesidad de informar a la sociedad sobre la otra cara de la energía nuclear y sus aportes positivos para el desarrollo de los países, para hacerlos más competitivos y amigables con el medio ambiente.

Palabras claves: Técnicas nucleares, isótopos, trazadores, radiofármacos, energía.

\section{INTRODUCCIÓN}

Es sabido que el Organismo Internacional de Energía Atómica (OIEA), es el promotor principal que impulsa cooperación científica y técnica en el uso pacífico de la tecnología nuclear. Cuenta con el apoyo de laboratorios especializados; así como también, con diversos programas de cooperación técnica de carácter regional y nacional. El uso de la tecnología nuclear ocurre en distintas áreas como lo son: Energía, alimentación y agricultura, salud, medio ambiente y aplicaciones industriales [1].

El objetivo de la mesa redonda titulada: "Técnicas nucleares y su versatilidad para la utilización y estudio de diversos procesos" es el de: Incentivar la aplicación de técnicas nucleares para realizar investigaciones y usar esos resultados para la generación de políticas basadas en evidencia. Esta actividad conto con la participación de expertos internacionales (Argentina y Brasil) y nacionales (IDIAP y UTP). Los temas tratados se enfocaron en los siguientes tópicos: Capacidad instalada para la investigación y uso de la tecnología nuclear, 
uso de isótopos en la investigación agrícola, uso de trazadores radioactivos para modelar procesos ambientales, la medicina nuclear y análisis estadístico en la investigación nuclear. El público asistente al evento estuvo conformado por: Investigadores internacionales y nacionales, profesores de universidad y secundaria, instituciones relacionadas con la salud, estudiantes de licenciatura realizado tesis como trabajo de grado.

\section{CONTENIDO}

La mesa redonda inició con la presentación "El papel del IPEN-CNEN/SP en la aplicación de la Tecnología Nuclear" de la Doctora Yasko Kodama, investigadora del Centro de Tecnologia das Radiações - CETER, del Instituto de Pesquisas Energéticas e Nucleares IPEN (Brasil). La Doctora Kodama, nos presentó un resumen de la capacidad instalada y de las líneas de investigación del Instituto para el cual labora en donde sobresalen aspectos como: La tecnología nuclear que se utiliza para mejorar las propiedades físicas y químicas de los materiales y reducir contaminantes indeseables, como patógenos o subproductos tóxicos, que el IPEN [2] es reconocido como una institución nacional líder en investigación y desarrollo en las áreas de radiofármacos, aplicaciones industriales de la radiación, diseño y operación de reactores nucleares y aplicaciones nucleares, ciencia y tecnología de materiales, tecnología láser y otras aplicaciones. Donde el CETER tiene un papel de importancia al trabajar, investigar y desarrollar nuevas tecnologías asociadas a los avances de otros campos de la aplicación de la tecnología nuclear como lo son: La preservación ambiental, radioesterilización, polímeros, nanotecnología, tomografía industrial, ciencia de los alimentos, biomateriales, preservación del patrimonio cultural, dosimetría, instrumentación nuclear.

La actividad continua con la intervención del Doctor José Villarreal del Instituto de Innovación Agropecuaria de Panamá y la presentación "Uso de Isotopos en la Investigación Agrícola y Ambiental", que abordó el tema del uso de isótopos para el estudio de diversos aspectos como: La eficiencia de la fertilización nitrogenada, la detección de la contaminación de acuíferos, discriminar contaminación por nitratos de origen urbano y ganadero, rastrear el movimiento y el origen de las emisiones de óxido nitroso, metano y dióxido de carbono en la agricultura, el estado hídrico de un cultivo, fertilidad de suelo y absorción de nutriente por las plantas, datación de monumentos o incluso rocas y materiales, estudios de calidad de agua, determinación de las pérdidas de suelo ocasionadas por la erosión y escorrentía, entre otros. En Panamá, a través de investigaciones realizadas en el IDIAP mediante proyectos de cooperación técnica con la Agencia Internacional de Energía Atómica (IAEA), se han hecho ensayos utilizando ${ }^{15} \mathrm{~N}$ para determinar la eficiencia de la fertilización nitrogenada en el cultivo de arroz, comprobando que esta oscila entre 20 y $35 \%$ tal como se había estimado por los métodos tradicionales. La relación ${ }^{13} \mathrm{C} /{ }^{12} \mathrm{C}$, se ha utilizado en ensayos con árboles 
de cacao para verificar como el cambio climático está afectando el suministro adecuado de agua a este cultivo ubicado en el bosque húmedo tropical. El ${ }^{114} \mathrm{Cd}$ se está empleando para estudiar la dinámica de la absorción del $\mathrm{Cd}$ en el árbol de cacao. Servirá para conocer que clones son menos acumuladores de Cd o cuales envían menor cantidad del metal de la raíz al fruto, beneficiando a los productores ya que la Unión Europea exige un máximo de 0,5 $\mathrm{mg}$ de $\mathrm{Cd} / \mathrm{kg}$ presente en el grano o fruto.

Posteriormente la Doctora Jimena Juri Ayub del Instituto de Matemática Aplicada San Luis (Argentina) abordó el tema "Uso de isotopos radiactivos como trazadores de procesos ambientales", presentando como elementos radiactivos presentes en el ambiente nos brindan una herramienta útil para evaluar y conocer distintos procesos que ocurren dentro del mismo. En los últimos años los procesos de degradación del suelo se han visto incrementados por el uso intensivo de las tierras y la expansión de la frontera agrícola por sobre formaciones vegetales naturales; entre otros factores. Por lo que es de vital importancia poder cuantificar el fenómeno, a fin de desarrollar medidas de conservación. Diferentes elementos radiactivos han demostrado ser una herramienta útil, rápida y económica para evaluar eficientemente y en forma conjunta tasas de erosión y sedimentación que ocurren dentro del paisaje $[3,4,5]$.

La Magíster Guadalupe González del Centro Regional de Chiriquí presenta el tema "Relación de las técnicas nucleares y la medicina actual"; por medio del cual, nos permite ver la importancia que tiene dentro de la Medicina, la tecnología nuclear, en aspectos como: El diagnóstico por imagen y tratamiento de enfermedades, el uso de radiofármacos que utilizan radionúclidos como el Tecnecio-99m, lodo-131, Fluor-18, entre otros. Así como, la importancia de destacar que las técnicas nucleares consideran los principios de protección radiológica para ser implementadas.

Finalmente, cierra las presentaciones la Doctora Nathalia Tejedor del Centro de Investigaciones Hidráulicas e Hidrotécnicas con el tema: "Aplicaciones del Análisis Estadístico de Datos en Técnicas Nucleares y Otras Investigaciones", presentación transversal cuyo propósito fue demostrar la importancia de analizar correctamente los datos, para interpretar y presentar los mejores resultados de nuestras investigaciones. En esta presentación se hizo énfasis en la utilidad que le proporciona un buen análisis estadístico al investigador de cualquiera de las disciplinas del conocimiento.

\section{CONCLUSIONES}

La mesa redonda titulada: "Técnicas nucleares y su versatilidad para la utilización y estudio de diversos procesos", fue de gran aceptación por los participantes debido a la variedad de los temas y el alcance dado por cada uno de los panelistas, se logró captar el interés de investigadores nacionales para establecer lazos de trabajo con los panelistas 
internacionales e incluso locales, los estudiantes aprovecharon la oportunidad de interactuar con los panelistas mediante sus preguntas a proyectos en específico, y sobre todo muy importante se instauro la inquietud de abordar otros temas de investigación de importancia dentro de los profesionales locales.

Parte de las preguntas que fueron realizadas hacia los panelistas fueron dirigidas en temas como: Agricultura (trazabilidad de las técnicas), cooperación internacional para la realización de ensayos sobre eficiencia de fertilización, uso de técnica analítica especifica para la determinación de radionucleidos, control de plagas por medio de esterilización usando irradiación, que tan seguros son los radiofármacos para la salud humana y posibilidad de fortalecimiento profesional en el tema de estadística descriptiva.

Nuestro principal resultado esperado y que estamos seguro de haber alcanzado con el desarrollo de esta sencilla pero importante actividad, fue de una sociedad (profesionales y estudiantes de nivel universitario) más receptiva en conocer los beneficios en la aplicación de las técnicas nucleares y sus alcances.

\section{Referencias}

[1] IAEA, «El OIEA en síntesis: atomos para la paz y el desarrollo» agosto 2018. [En línea]. Available: https://www.iaea.org/sites/default/files/19/01/iaeaataglance_sp.pdf. [Último acceso: julio 2021]

[2] IPEN, «Centros de Pesquisa» 2021. [En línea]. Available: https://www.ipen.br/portal_por/portal/centros. php?secao_id=27. [Último acceso: maio 2021]

[3] Lohaiza F, Velasco H, Juri Ayub J, et al. Annual variation of 7Be soil inventory in a semiarid region of central Argentina. J Environ Radioact. 2014;130:72-77. http://dx.doi.org/10.1016/i. ienvrad.2014.01.006

[4] de Rosas JP, Esquivel AD, Heimann DM, et al. Using beryllium-7 to evaluate soil erosion processes in agricultural lands in semiarid regions of Central Argentina. Environ Earth Sci. 2018;77(16):587. https://doi.org/10.1007/s12665-018-7767-x

[5] Esquivel AD, Moreira RM, Monteiro RPG, et al. Quantification of soil erosion using ${ }^{7} B e$ in a steep watershed used for natural grazing in Brazil. Isot. Environ. Health Stud. 2021;57(3):316-331. https:// doi.org/10.1080/10256016.2021.1918687

\section{Autorización y Licencia CC}

Los autores autorizan a APANAC XVIII a publicar el artículo en las actas de la conferencia en Acceso Abierto (Open Access) en diversos formatos digitales (PDF, HTML, EPUB) e integrarlos en diversas plataformas online como repositorios y bases de datos bajo la licencia CC:

Attribution-NonCommercial-ShareAlike 4.0 International (CC BY-NC-SA 4.0) https://creativecommons.ora/ licenses/by-nc-sa/4.0/.

Ni APANAC XVIII ni los editores son responsables ni del contenido ni de las implicaciones de lo expresado en el artículo. 